\title{
Advanced Abdominal Ectopic Pregnancy with Subsequent Fetal and Placental Extraction: A Case Report
}

\author{
Luis Armando Zuñiga ${ }^{a}$ César Alas-Pineda ${ }^{b}$ Clarisa L. Reyes-Guardadoc \\ German Isaías Melgar ${ }^{a}$ Kristhel Gaitán-Zambranoc Simmons Gough ${ }^{\text {d }}$ \\ ${ }^{a}$ Departamento de Ginecología y Obstetricia, Hospital Dr. Mario Catarino Rivas, San Pedro Sula, Honduras; \\ ${ }^{b}$ Departamento de Epidemiología, Hospital Dr. Mario Catarino Rivas, San Pedro Sula, Honduras; ${ }^{C} F a c u l t a d ~ d e$ \\ Medicina y Cirugía, Universidad Católica de Honduras - Campus San Pedro y San Pablo, San Pedro Sula, Honduras; \\ 'Departamento de Imagenología y Radiología, Hospital Civil de Guadalajara Fray Antonio Alcalde, Guadalajara, \\ Mexico
}

\section{Established Facts}

- Abdominal ectopic pregnancy (AEP) incidence varies from 1:10,000 to 1:30,000 pregnancies worldwide.

- AEP is an important cause of maternal and fetal death with high mortality rates.

\section{Novel Insights}

- This case represents a clinical challenge, especially in terms of surgical management for placental extraction.

- Although newborn survival mortality rates account for $40-95 \%$ of the cases, the neonate was born without complications.

\section{Keywords}

Abdominal pregnancy $\cdot$ Ectopic pregnancy $\cdot$ Case report

\section{Abstract}

Abdominal ectopic pregnancy (AEP) occurs within the peritoneal cavity, outside the genital organs (uterus, tubes, ovaries). It is an unusual condition with an incidence that varies from 1:10,000 to $1: 30,000$ of all pregnancies worldwide. A 38-year-old primigravid patient was diagnosed in the sec- ond trimester with AEP. Pregnancy reached 35.6 gestational weeks, and the patient underwent surgery via laparotomy for extraction of the live fetus. Complete removal of the placenta was performed without maternal or fetal complications. AEP is an important cause of maternal and fetal death; the mortality rate in pregnant women with AEP is approximately $1-18 \%$. Surgical intervention to deliver a baby in cases of AEP requires a multidisciplinary team, especially in countries with limited therapeutic options.

(C) 2022 The Author(s).

Published by S. Karger AG, Basel
Karger@karger.com www.karger.com/bmh

Karger $\stackrel{\text { ' }}{5}$

BOPEN ACCESS
(C) 2022 The Author(s)

Published by S. Karger AG, Basel

This is an Open Access article licensed under the Creative Common Attribution-NonCommercial-4.0 International License (CC BY-NC) (http://www.karger.com/Services/OpenAccessLicense), applicable to the online version of the article only. Usage and distribution for commercial purposes requires written permission. 


\section{Introduction}

Ectopic pregnancy (EP) is an obstetric complication affecting women of reproductive age and is defined as the extrauterine implantation of a fertilized egg. It occurs in approximately $1.2-1.4 \%$ of all reported pregnancies worldwide [1].

There are multiple risk factors that predispose patients to EP, including a history of pelvic inflammatory disease, smoking, fallopian tube surgery, previous EP, endometriosis, assisted reproduction techniques, and infections $[2,3]$. However, only $50 \%$ of women with abdominal EP (AEP) have any associated risk factors [4].

Ninety percent of EPs are tubally located, while the rest are distributed in cesarean scars (4\%), interstitial regions (4\%), intramural regions (1\%), the myometrium (1\%), and the abdominal cavity (1.3\%) [5].

AEP is rare and occurs within the peritoneal cavity outside of the genital organs (uterus, tubes, ovaries) [6]. Its incidence varies from $1: 10,000$ to $1: 30,000$ pregnancies worldwide $[6,7]$. AEP is usually diagnosed late because some patients are asymptomatic, or if symptomatic, the symptoms are nonspecific [8]. It may present as diffuse pain accompanied by signs of incipient pregnancy [9].

Depending on its location, AEP can be classified into two types: primary, which occurs when fertilization and implantation occur directly in the abdominal organs [3]; and secondary, which occurs as a result of a tubal pregnancy that detaches into the abdominal cavity (tubal abortion, rupture of a uterine horn, or a caesarean section scar) [10]. Other, more unusual cases such as the iatrogenic perforation of the uterus during a voluntary termination of pregnancy may also occur $[10,11]$.

AEP can reach full-term gestation, with a viable fetus and subsequent perinatal survival; however, these are exceptional cases described in the literature [6]. In Honduras, there is a record of only one previous case of AEP in 1956, with a dead fetus that developed into a lithopedion [12]. The relevance of the case described below lies not only in the rarity of AEP but also in the procedure performed and the results obtained in a third-level hospital in San Pedro Sula, Honduras, C.A., where the first total placental extraction performed in the AEP resulted in a live, healthy baby.

\section{Case Report}

We present the clinical case of a 38-year-old primigravid woman, who was sexually active since she was 15 years old and did not use family planning methods. Her obstetric history includes un-

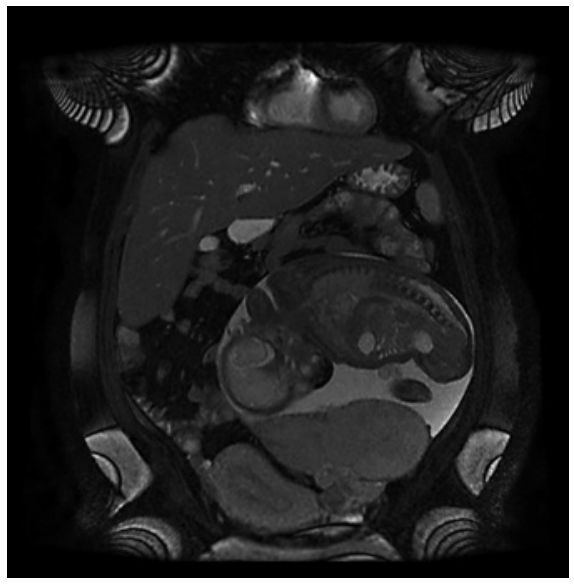

Fig. 1. Coronal T2 section of an abdominopelvic MRI showing the amniotic sac and the fetus in the abdominal cavity attached to the left adnexa and displacing the empty uterus to the right.

treated polycystic ovaries and irregular menstruation with cycles from 28 to 40 days.

In July 2020, the patient sought medical care for amenorrhea that was observed since March of the same year. She also reported that she took two home pregnancy tests (urine) and a blood pregnancy test during March, all of which were positive.

A pelvic ultrasound (US) was performed, which revealed an empty, homogeneous, and regularly contoured uterus. She was referred to Hospital Dr. Mario Catarino Rivas (HMCR) in San Pedro Sula, for management of her extrauterine pregnancy. However, the patient refused the referral and subsequent evaluations at HMCR, alleging that the hospital would terminate her pregnancy and that her desire was to continue with the pregnancy. In addition to this argument, the patient also stated that she was afraid that she might contract COVID-19.

The following month, at approximately 20 gestational weeks (GWs) according to her last menstrual period, the patient again sought medical care for her pregnancy. A pelvic US was performed and she was referred for the second time to HMCR. The patient finally agreed and on the following day, i.e., August 13, 2020, she went to HMCR. Because she was diagnosed with AEP, she was admitted for further evaluation and a complete blood test was performed, which was normal. Further, the patient did not report abdominal pain.

Physical examination revealed that the patient was in good general condition with a mesomorphic biotype. Her vital signs were stable, with a blood pressure of $120 / 80 \mathrm{~mm} \mathrm{Hg}$, a heart rate of 72 beats per minute, a respiratory rate of 19 breaths per minute, and a normal body temperature. Segmental physical examination showed no apparent abnormalities. Positive fetal movement and a fetal heart rate of 150 beats per minute were found upon examination of the fetus.

A US performed after admission revealed that the placenta in the left hemiabdomen adhered to the left abdominal wall and the left pelvic wall, with color Doppler flow in close contact with the uterus at the level of the left adnexa and left uterine artery. These findings were confirmed with magnetic resonance imaging (MRI), 

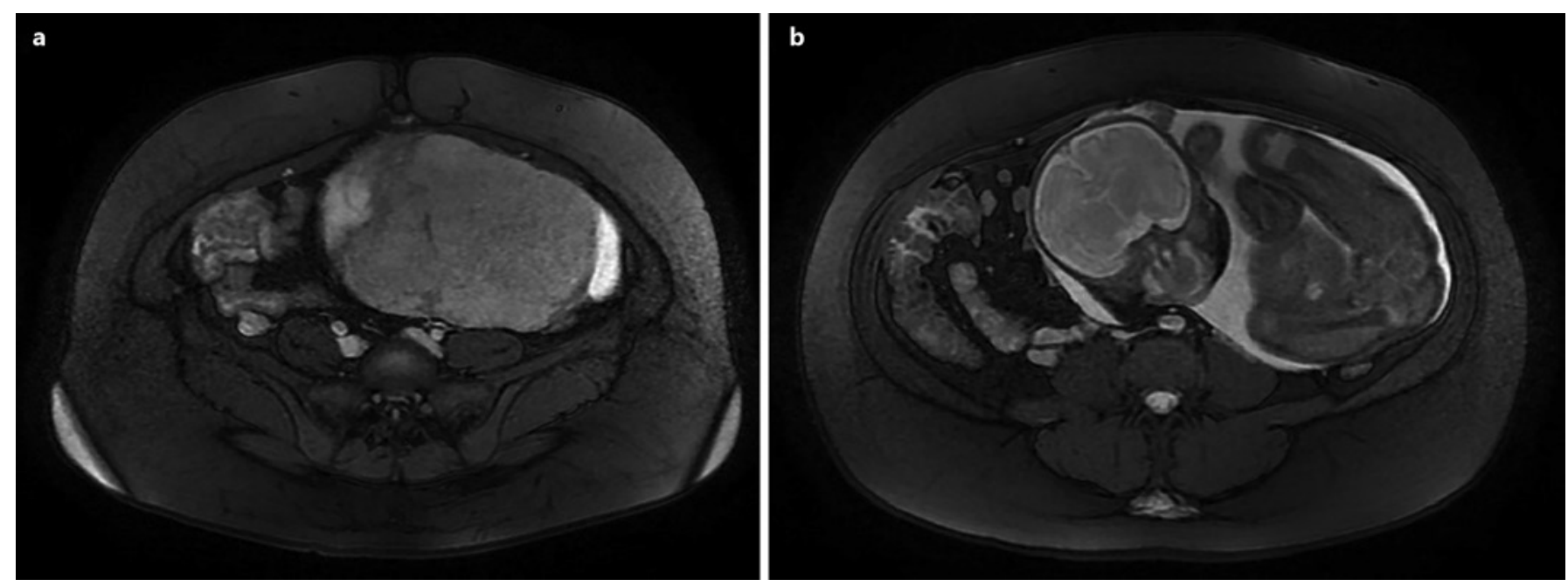

Fig. 2. a Axial T2 section of an abdominopelvic MRI showing the amniotic sac and the placenta without a separation plane between it and the anterior abdominal wall. b A more cephalic view showing the fetus in the abdominal cavity displacing intestinal viscera to the right.

performed the same day, where the presence of intraperitoneal extrauterine pregnancy was also observed in the left hemiabdomen (shown in Fig. 1, 2). The primigravid woman was informed of the situation and possible outcome of the diagnosis, emphasizing the maternal-fetal complications and risks. The patient left the health facility the same day, fearing that the pregnancy would be terminated.

In September, she returned to the hospital for evaluation, where an abdominal US found no significant changes relative to the previous one. She was asked to remain in the facilities for surveillance and follow-up; however, she again refused hospitalization and left HMCR.

The patient was hospitalized at HMCR on November 2nd of the same year, at 31.6 GW. She returned because she researched her diagnosis on the internet and became concerned when she read about the high maternal and fetal mortality rates associated with AEP. The patient was asymptomatic when admitted to the hospital. The patient was treated with fetal lung maturation inducers and dexamethasone in preparation for the delivery of the fetus.

A third US revealed a single fetus in the abdominal region, with an estimated weight of 2,144 $\mathrm{g}$ and an amniotic fluid index of 10 $\mathrm{cm}$. The patient decided to continue the pregnancy at $33 \mathrm{GW}$, despite the risks. The patient consented to a laparotomy to deliver the fetus on December 2, 2020.

The surgery was initially planned to be performed at $36 \mathrm{GW}$; however, when the multidisciplinary team was formed, it was agreed to perform the surgery at $35.6 \mathrm{GW}$ because the entire team would be present. The team consisted of two obstetrician gynecologists, one general surgeon, two urologists, two pediatricians, three anesthesiologists, one gynecology resident, nursing staff, and a medical instrument specialist. In preparation for the operation, space was reserved in the neonatal and adult intensive care units and a supply of blood products was secured from the hospital's blood bank.
Prior to surgery, a double-J stent was placed in both ureters. Under spinal anesthesia, a medial laparotomy was performed, with an infraumbilical and supraumbilical incision of approximately 25 $\mathrm{cm}$, which revealed amnion-omental adhesions. The placenta was adhered to the left abdominal wall, with several firm points of attachment to the left uterine adnexa. The right ovary was of normal appearance and morphology. Amnion-omental adhesions were released, resulting in the discharge of a copious amount of amniotic fluid that was colorless, not foul smelling, and at normal body temperature.

The fetal membranes were identified, and the fetus, which was in a transverse dorso-anterior position, was extracted (shown in Fig. 3a). This resulted in the birth of a live male to whom late clamping of the umbilical cord was applied. The newborn was evaluated by a pediatrician and taken to the neonatal nursery in good health condition. The patient was administered 1 vial of tranexamic acid $(1,000 \mathrm{mg} / 10 \mathrm{~mL})$ and a $20 \mathrm{~mL}$ vial of intravenous human prothrombin complex $\left(\right.$ Octaplex $\left.^{\circledR}\right)$. The location and vascularization of the placenta were evaluated transoperatively, and a manual placental extraction was performed.

Complete placental extraction was achieved by digital separation between the placenta and the anterior abdominal wall. Surgical scissors were used to separate the colon from the placenta, causing lacerations of the colonic serosa in that portion. Removal of the left adnexa, to which a portion of the placenta was firmly adhered, was performed because it was the main source of blood supply for the placenta (shown in Fig. 4). During the procedure, the patient lost approximately 2,000 $\mathrm{mL}$ of blood, for which two units of packed red blood cells and four units of fresh frozen plasma were required.

The procedure ended without complications, and the patient was hemodynamically stable with normal vital signs. The patient was transferred to surgical recovery and then to the puerperium room to remain under medical surveillance for 2 days after the procedure with no complications. The newborn male was in good 
Fig. 3. a Extraction of the fetus $35.6 \mathrm{GW}$. b The live and healthy male newborn.
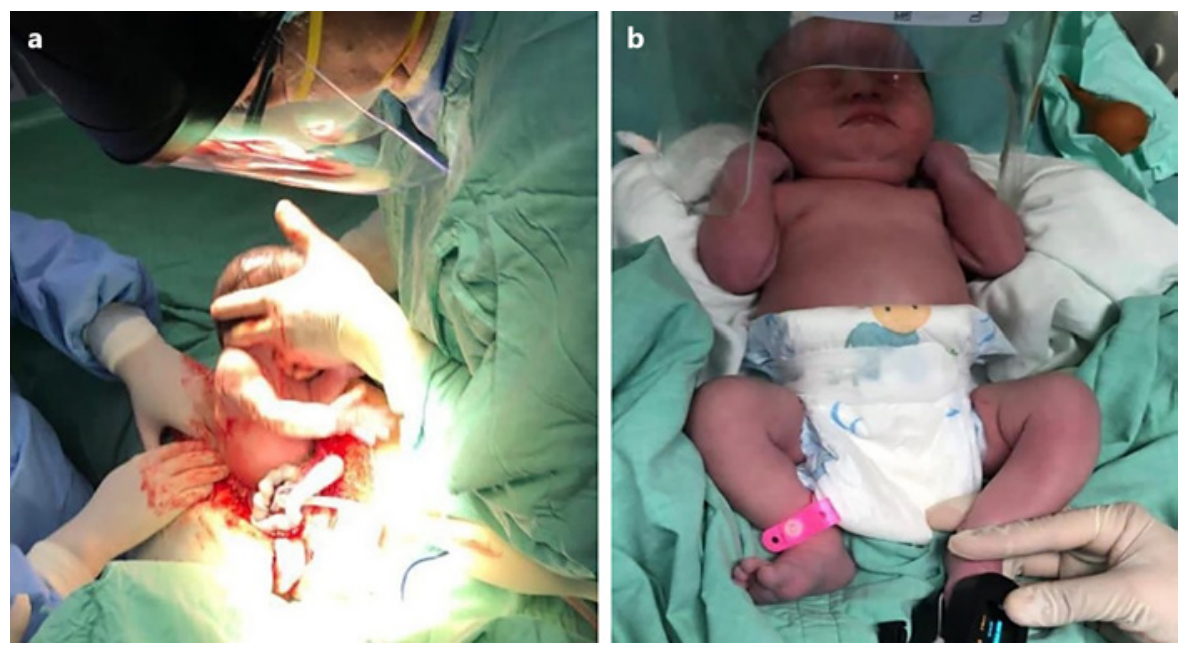

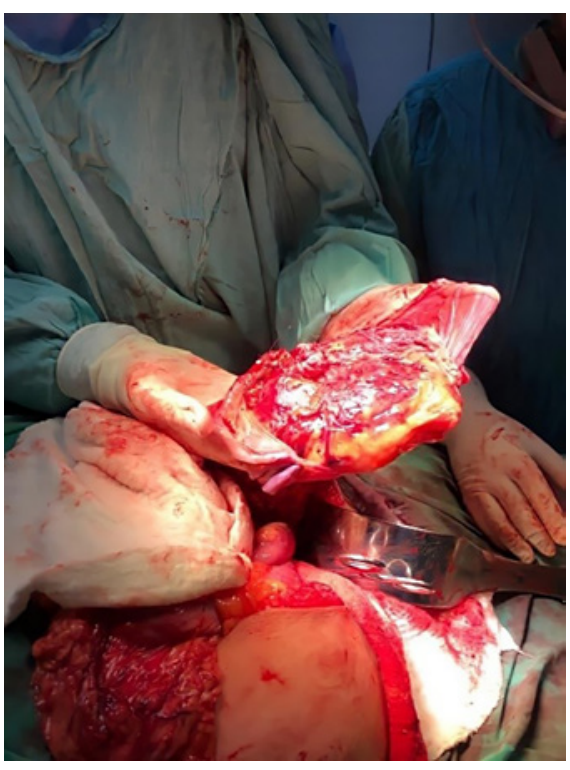

Fig. 4. Complete removal of the adhesion from the abdominal and pelvic cavity.

health, with an APGAR score of 8/9, weighed 2,640 g, was $46 \mathrm{~cm}$ long, and had a head circumference of $33 \mathrm{~cm}$. The baby had no apparent congenital or structural malformations (shown in Fig. 3b).

\section{Discussion}

$\mathrm{AEP}$ is an unusual variant of EP, which is characterized mainly by nesting and embryonic growth in the peritoneal cavity [3]. The most frequent sites of placental implantation are the posterior cul-de-sac, which accounts for $55 \%$ of cases, followed by the mesosalpinx, the omentum, the peritoneum of the abdominal or pelvic walls, and the space between the anterior uterine wall and the bladder [13-15]. According to the worldwide literature, from 2008 to 2013 , only 38 cases of advanced abdominal pregnancy resulting in a live birth were reported $[10,16]$.

AEP is an important cause of maternal-fetal death; the mortality rate in pregnant women ranges from approximately $0.5-18 \%$, exceeding $7-8$ times the mortality that occurs in tubal EP and is 90 times higher than the mortality in normal intrauterine pregnancy $[3,14,17]$. This is due to the high risk of profuse hemorrhage due to partial or total placental separation [15].

Newborn survival is also affected, with a perinatal mortality rate of $40-95 \%$, and $21 \%$ of live-born infants have birth defects [18]. Typical deformities include limb defects, facial and cranial asymmetry, joint abnormalities, and central nervous system malformations [18]. The neonate had no malformations, no perinatal complications, remained under surveillance, and was subsequently discharged.

Primary AEP is an unusual obstetric complication of EP located in the peritoneal cavity, with an incidence of approximately $0.6-1.6 \%$ of all cases reported in the literature [19]. The patient was diagnosed with primary AEP and was the first case of this type reported in Honduras.

Currently, the criteria established by Studifford in 1942 are used to define primary abdominal EP, which include the following: (1) normal bilateral fallopian tubes and ovaries without the presence of recent or past lesions, (2) absence of utero-peritoneal fistula, and (3) pregnancy in exclusive contact with the peritoneal surface [20]. 
AEP beyond the second trimester with a viable fetus is extremely rare. However, it is the only form of EP that can result in a fetus fit for extrauterine life. There are few cases of full-term abdominal gestation described in the world literature [8]. Despite the risks, the patient held firm to her religious beliefs, and since it was her first pregnancy, decided to continue with the pregnancy even after the diagnosis of AEP.

Due to abnormal placental implantation, cases of advanced abdominal pregnancy carry multiple risks such as hemorrhage, infection, disseminated intravascular coagulation, abdominal obstruction, fistula formation, and preeclampsia $[13,16,18,19]$. In the case described herein, there were no complications during the gestational, transoperative, or postoperative period.

Clinical evaluation is important to diagnose an abdominal pregnancy, unlike tubal EPs which may go undetected until late gestational age [9]. This is due to a clinical picture that varies from asymptomatic patients to others with severe symptoms that can compromise both maternal and fetal life [3].

Monitoring serum beta-human chorionic gonadotropin levels is a useful marker for clinical suspicion of EP; however, it is not sufficient by itself to make the diagnosis of EP. In many cases, the diagnosis of AEP is not confirmed until a laparotomy or laparoscopy is performed due to suspicion of a tubal EP [3, 10, 14, 19, 21].

Diagnostic imaging methods include MRI and US. The latter can reach a sensitivity of $99 \%$ if used properly $[13,19,21]$. When observed during a US, an empty uterus with gestational sac separated from the uterus, adnexa, and ovaries should raise suspicion of AEP. A mass seen in the abdomen with features of pregnancy (gestation and yolk sacs, fetal heartbeat) is diagnostic, but it is usually not possible to differentiate a pelvic mass from the adnexa using US [3]. MRI offers better visualization of the placenta, uterus, and fetal tissues [13]. To confirm the location of placental and fetal tissue, MRI was used.

Surgery is the preferred procedure for AEP, and the best option is to remove the entire sac including fetus, membranes, and placenta [19]. Laparotomy is the preferred method in cases where there is a risk of hemorrhage; laparoscopic surgery may be chosen in cases that are diagnosed early and do not carry vascular risks [14, 21].

Placental extraction carries the risk of uncontrollable hemorrhage. It is not considered as the first treatment option for AEP because it lacks the hemostatic mechanisms exerted by myometrial contractions; therefore, it fails to constrict the placental vasculature [13]. Despite the risks involved in placental extraction, the multidisciplinary team made the decision to remove it and proceeded to perform digital separation between the placenta and the anterior abdominal wall without further complications. This caused moderate bleeding, which was treated by promptly ligating the blood vessels that supplied the placenta.

The general recommendation is to leave the placenta in situ to reduce the risk of severe perioperative hemorrhage; however, this treatment has been associated with postoperative mortality and complications. These include secondary hemorrhage, abscess formation, adhesions, coagulopathies, ongoing preeclampsia, and failure of lactogenesis $[13,14,16,19,22]$. When the placenta remains in situ, two methods of follow-up are available [13]. The first is the use of methotrexate to accelerate tissue absorption, and the second method is expectant management, which consists of monitoring the beta-human chorionic gonadotropin levels and performing a US [13].

\section{Conclusion}

Full-term AEP with subsequent placental abruption is an extremely rare situation in clinical practice. Early diagnosis provides a better prognosis for the mother; however, it may go unnoticed due to its clinical variability. Surgical treatment is the method of choice for these cases, despite the high risks associated with full-term gestation including high maternal-fetal mortality rates. There is no consensus on the approach, and some authors recommend leaving the placenta in situ, despite the postoperative complications that this entails. In cases in which placental extraction is performed, a good separation technique and adequate vascular management will provide the best results.

\section{Statement of Ethics}

According to the guidelines for case reports of the Universidad Católica de Honduras Ethics Committee, this study is exempt from requiring ethics approval. The study was performed ethically in compliance of the Helsinki Declaration. Written informed consent was obtained from the patient for publication of this case report and any accompanying images.

\section{Conflict of Interest Statement}

The authors have no conflicts of interest to declare.
Zuñiga/Alas-Pineda/Reyes-Guardado/ Melgar/Gaitán-Zambrano/Gough 


\section{Funding Sources}

This research received no specific grant from any funding agency in the public, commercial, or not-for-profit sectors.

\section{Author Contributions}

Luis Armando Zuñiga, diagnosed, treated, analyzed, and interpreted the patient's clinical information and the monitoring and conceived the idea; Clarisa L. Reyes-Guardado coordinated and worked on data collection. Kristhel Gaitán-Zambrano, Clarisa L. Reyes-Guardado, and German Isaías Melgar wrote the paper. César Alas-Pineda and Luis Armando Zuñiga reviewed and edited the final draft. All authors critically reviewed and approved the final draft and are responsible for the content and similarity index of the manuscript. All authors read and approved the final manuscript.

\section{Data Availability Statement}

Patient's files and datasets used to support the findings of this study are restricted to protect the privacy of clinical data. Data are available to investigators who comply the criteria for access to confidential data under request. Requests for access to these data should be directed to César Alas-Pineda: cesar_alas10@hotmail. com.

\section{References}

1 Rana P, Kazmi I, Singh R, Afzal M, Al-Abbasi FA, Aseeri A, et al. Ectopic pregnancy: a review. Arch Gynecol Obstet. 2013 Oct;4(288): 747-57.

2 Hendriks E, Rosenberg R, Prine L. Ectopic pregnancy: diagnosis and management. Am Fam Physician. 2020 May 15;101(10):599606.

3 Gutiérrez Y, Alvaro A, Campillo Meza J, Garrido Fernandez P, Rodriguez B, Castán Mateo S. Embarazo ectópico abdominal. Diagnóstico y tratamiento médico con metotrexato. Prog de Obstet y Ginecol. 2011 May;54(5): 257-60.

4 Kopelman ZA, Keyser EA, Morales KJ. Ectopic pregnancy until proven otherwise ... even with a negative serum hCG test: a case report. Case Rep Womens Health. 2021 Apr;30(1): e00288.

5 Panelli DM, Phillips $\mathrm{CH}$, Brady PC. Incidence, diagnosis and management of tubal and nontubal ectopic pregnancies: a review. Fertil Res Pract. 2015;1(1):15.

6 Yoder N, Tal R, Martin JR. Abdominal ectopic pregnancy after in vitro fertilization and single embryo transfer: a case report and systematic review. Reprod Biol Endocrinol. 2016 Oct 19;14(1):69.

7 Mengistu Z, Getachew A, Adefris M. Term abdominal pregnancy: a case report. J Med Case Rep. 2015 Jul 28;9(9):168.
8 Rahaman J, Berkowitz R, Mitty H, Gaddipati S, Brown B, Nezhat F. Minimally invasive management of an advanced abdominal pregnancy. Obstet Gynecol. 2004;103(1):1064-8.

9 Siati A, Berrada A, Kharbach A. Abdominal pregnancy with a healthy newborn: a new case report. Pan Afr Med J. 2019 Sep;34(1):35.

10 Aragon-Charry J, Santos-Bolíva J, TorresCepeda D, Reyna-Villasmil E. Embarazo ectópico primario en epiplón. Reporte de caso. Prog de Obstet y Ginecol. 2014 Jan;57(1):302.

11 Hernández Núñez J, Abreu Díaz A, Michael Ndwambi N. Ectopic abdominal pregnancy due to uterine perforation after an attempt to terminate pregnancy: a case presentation. Medwave. 2017 Jul 17;17(6):e7000.

12 Faraj E. Un caso de embarazo abdominal. Rev Méd Hond. 1958 Oct 3;26(4):107-14.

13 Mora-Enríquez JA, Arreaza-Graterol M, Nossa-Moreno HH, Rodríguez-Ortiz JA. Embarazo abdominal avanzado: diagnóstico y manejo. Reporte de un caso y revisión de la literatura. Rev Colomb Ginecol Obstet. 2014 Jul-Sep;65(3):256-61.

14 Adesiyun AG, Audu AI. Term extrauterine pregnancy in a Nigerian mother: a case report and review of the literature. Arch Gynecol Obstet. 2009;279(1):75-7.

15 Yildizhan R, Kolusari A, Adali F, Adali E, Kurdoglu M, Ozgokce C, et al. Primary abdominal ectopic pregnancy: a case report. Cases J. 2009;2(8):84-5.
16 Masukume G, Sengurayi E, Muchara A, Mucheni E, Ndebele W, Ngwenya S. Fullterm abdominal extrauterine pregnancy complicated by post-operative ascites with successful outcome: a case report. J Med Case Rep. 2013 Jan;7(7):10.

17 Zhang J, Li F, Sheng Q. Full-term abdominal pregnancy: a case report and review of the literature. Gynecol Obstet Invest. 2008;65(2): 139-41.

18 Gurjar R. Full-term live secondary abdominal pregnancy: a rare case report. J Obstet Gynaecol India. 2019 Apr;69(1):36-9.

19 Sheng-Wen S, Jenn-Jeih H, Ho-Yen C, ChienMin H, Fang-Chun C, Yao-Lung C, et al. Management of primary abdominal pregnan$c y$ : twelve years of experience in a medical centre. Acta Obstet Gynecol Scand. 2007; 86(1):1058-62.

20 Hailu FG, Yihunie GT, Essa AA, Tsega WK. Advanced abdominal pregnancy, with live fetus and severe preeclampsia, case report. BMC Pregnancy Childbirth. 2017;17(1):243.

21 Hajji A, Toumi D, Laakom O, Cherif O, Faleh R. Early primary abdominal pregnancy: diagnosis and managment. A case report. Int J Surg Case Rep. 2020;73:303-6.

22 Dahab AA, Aburass R, Shawkat W, Babgi R, Essa O, Mujallid RH. Full-term extrauterine abdominal pregnancy: a case report. J Med Case Rep. 2011 Oct;5(211):531. 